\title{
Famous brain set to go under the knife
}

\section{Slices from the brain of H.M., a key patient in pioneering memory studies, will be immortalized online.}

Neuroanatomist Jacopo Annese will next week begin slicing one of the most precious pieces of tissue in the history of neuroscience: the brain of the famous amnesiac Henry Gustav Molaison, more commonly known by his initials, H.M.

In 1953, Molaison underwent an experimental operation that aimed to treat his severe epilepsy, during which the surgeon removed a part of his brain, including a large chunk of the hippocampus. For the rest of his life, Molaison's ability to form new memories was severely impaired, although he could easily recall memories from his childhood. Hundreds of ensuing psychological studies on him have yielded invaluable insight into memory formation, the separation of different cognitive functions, and the relationship between brain structure and function.

On 2 December, exactly one year after Molaison's death, Annese, of the University of California, San Diego, will begin dividing the brain into roughly 2,400 slices, each thinner than a human hair, and digitizing them. Annese hopes that Molaison's brain will become the first of many in a digital human-brain library at the university.

Annese is one of the few people with the sophisticated equipment needed to slice whole human brains, which is how he came by Molaison's brain. Most labs cut human brains into blocks before slicing them - the fate that befell Albert Einstein's brain.

Annese will mount and stain about every 30th slice for cell nuclei and projections, which will allow him to map the cellular architecture in three dimensions. The remaining slices will be available to the neuroscience community, with researchers able to view the particular slice they want to study before requesting it.

Because demand for certain regions of Molaison's brain is likely to outstrip supply, Annese plans to create a selfmoderating forum through which scientists can discuss how to distribute the pieces. "Everyone will be aware that a certain person has this piece of tissue," he says. "It will be their own prerogative for their reputation to do something valuable with it."

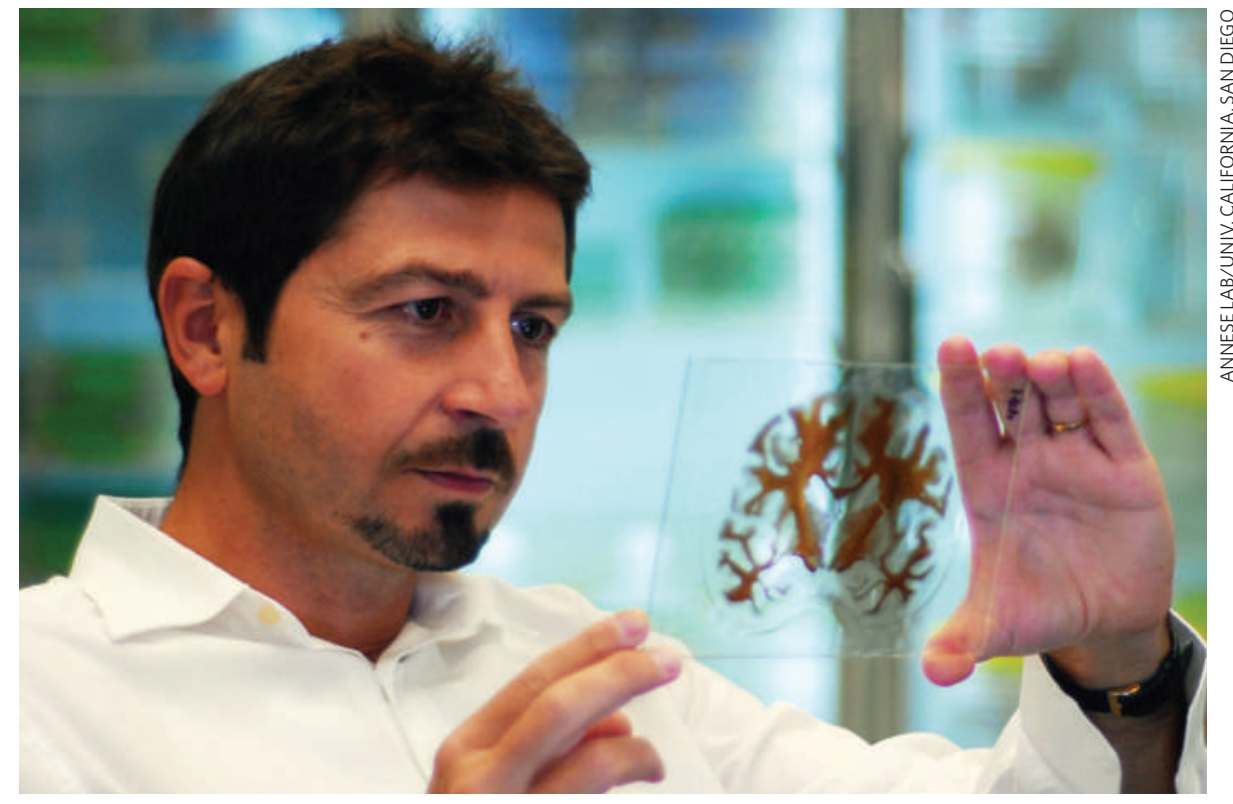

Jacopo Annese hopes to make thousands of slides and create a three-dimensional brain map.

The tissue will give neuroscientists who worked with Molaison a chance to test hypotheses through detailed anatomical study.

"People can finally look in detail at the precise border of the [brain] lesion, and what the consequences were," says Sandra Witelson at the Michael G. DeGroote School of Medicine at McMaster University in Hamilton, Canada, who studied Einstein's brain and oversees the world's largest repository of normal human brains.

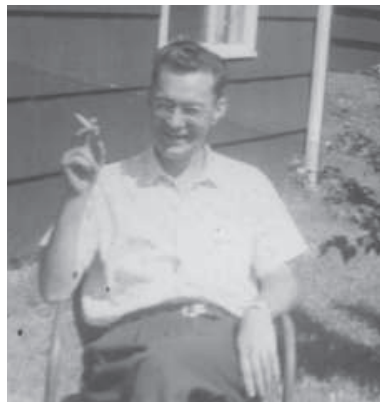

"After an experimental operation to treat his epilepsy, Molaison's ability to form new memories was severely impaired."
If certain hippocampal structures remained intact, this might explain why Molaison could sometimes recall the names of people who became famous after his injury, such as presidents John F. Kennedy and Ronald Reagan. Studies may also show whether Molaison developed Alzheimer's disease and could hint at whether other brain areas became stronger to compensate for his injury.

Suzanne Corkin, a behavioural neuroscientist at the Massachusetts Institute of Technology in Cambridge who worked with Molaison for 46 years, is "ecstatic" about Annese's project. She says she is looking forward to the chance for others to test their hypotheses against the physical material from Molaison.

But even when accompanied by a mountain of clinical analyses, there is only so much that can be learned from a single brain. "There are still some lingering questions about H.M., but I'd expect that most of what we can learn from him we've already learned," says Adam Gazzaley, a cognitive neuroscientist at the University of California, San Francisco.

To help extend the findings from Molaison's brain, Annese wants to slice, digitize and disseminate many other brains, including some from other people with memory problems. He has already processed the brain of a patient who had some similar cognitive deficits to Molaison after his hippocampal area was damaged by a viral infection.

Annese hopes that the publicity surrounding Molaison's case will bring attention and funding to the brain-library initiative, helping him to overcome the biggest hurdle: storing the data. To make it possible to zoom in on one slide of brain from life size to the cellular level requires 20,000 images, he says - which will require $1-10$ terabytes per slide.

He wants to provide 500 or so such slides for each of hundreds of brains.

Lizzie Buchen 